\title{
DAS BARBARENBILD DES POSEIDONIOS UND SEINE STELLUNG IN DER PHILOSOPHISCHEN TRADITION
}

The author gives an account of the ideas of the Greeks (Herodot, Hippocrates, Plato, Aristotle) about the barbarians. They hesitate between admiring some barbarians, or, on the contrary, considering them unvalued people in comparison with the Greeks, and the theory of the climate, which would be definitive on the formation of the men's bodies and souls.

Poseidonios discusses this problem in a well known fragment in which he tells how the Mariandins submitted to Heraclea because they found themselves inferior. We can come across with traces of his thought on Cicero, De re publica III and Strabo, VI. In conclusion, Poseidonios justifies the Roman world power because its superiority, and this because the climate of Italy. However, he insists that this power must be used with justice and blames the bloody actions of the Sicilian slave war.

Nicht nur als Faktor der politischen, wirtschaftlichen und gesellschaftlichen Entwicklung hat das Verhältnis von Zentrum und Peripherie in der antiken Welt eine bedeutsame Rolle gespielt. Wichtig waren auch seine Reflexe im geistigen und kulturellen Leben, die ihrerseits zu einer Triebkraft im historischen Prozeß wurden. Neben Dichtung und bildender Kunst bewiesen Philosophie und Wissenschaft in diesem Zusammenhang eine nachhaltige Wirkung. Wesentliche Handlungsmotivationen der praktischen Politik in der Geschichte Griechenlands und Roms werden nur dann verständlich, wenn man das gesellschaftlich-politische Weltbild erfaßt, in das auch das Verhältnis von Zentrum und Peripherie eingeordnet war. Die Begriffspaare Hellenen-Barbaren und RömerBarbaren gehören zu den Grundkoordinaten, die Denken und Handeln in der Antike über Jahrhunderte mit bestimmt haben. In römischer Zeit erfährt die Auseinandersetzung mit diesem Problem noch einmal eine überaus bemerkenswerte Wendung bei dem herausragenden Philosophen, Gelehrten und Historiker Poseidonios. Diese gibt einerseits eine Antwort auf Fragen, die sich mit der historischen Entwicklung in der Endphase der römischen Republik neu stellen. Sie ist andererseits nur verständlich aus dem Verhältnis des Poseidonios zu dem vorgeprägten 
Gedankenmaterial einer langen Tradition. Diese müssen wir zunächst skizzieren, bevor wir uns Poseidonios zuwenden.

Das Barbarenbild der antiken Philosophie und Wissenschaft ist eine äußerst komplexe Erscheinung '. Vielfältige und z. T. auch sehr widersprüchliche Tendenzen treffen und kreuzen sich von den Anfängen geographisch-ethnographischer Forschung im 6. Jh. v. Chr. bis in hellenistisch-römische Zeit. Das Bestreben der ionischen iбторí, von den der antiken Kultur nicht oder nicht unmittelbar zugehörigen Völkern ein möglichst objektives Bild zu vermitteln, bleibt in starker Spannung zu anderen Tendenzen in der ganzen Antike lebendig: bei den Ethnographen, bei den Historikern und bei den Philosophen ${ }^{2}$. Schon früh wird eine Neigung spürbar, die sich als Tendenz durch die gesamte Antike zieht: bestimmte Barbarenvölker, besonders solche des fernen Nordens und Südens zu idealisieren und bisweilen diese Vorläufer des "bon sauvage' der neuzeitlichen Aufklärung sogar zum Träger sozialutopischer Konzeptionen werden zu lassen. Eine weitere Hauptrichtung stand im Dienste der Begründung politischer Herrschaftstheorie. Hier geht es um die Hellenen-Barbaren-Antithese im klassischen Sinn: um die Begründung weitreichender Herrschaftsansprüche, um die Rechtfertigung expansiver Politik, um die theoretische Fundierung der Sklaverei. Unter den beträchtlichen Schwierigkeiten, die mit dem Versuch verbunden sind, eine über Jahrhunderte sich erstreckende Entwicklung in knappen Strichen zu umreißen, ist nicht die geringste die außerordentliche Komplexität, mit der auch diese dritte Hauptlinie in Erscheinung tritt. Ist sie doch mannigfach überlagert von divergierenden Tendenzen bei einem und demselben Philosophen, Fachgelehrten oder Historiker.

Der monumentale Satz am Eingang des Herodoteischen Geschichtswerkes umreißt ein Programm universalgeschichtlicher Dimension, im Werk realisiert vor allem mit den Logoi über Lyder, Perser, Babylonier, Ägypter, Skythen, Libyer und Thraker, von vielen kleineren Exkursen abgesehen. Bei der Frage nach den Ursachen für den welthistorischen Konflikt zwischen Asien und Europa verweist der Historiker später auf

I Aus der umfänglichen Literatur zum Thema können hier nur einige neuere Arbeiten genannt werden: Grecs et barbares. Fondation Hardt. Entretiens sur l'antiquité classique VIII, Vandoeuvres-Genève 1962; H. C. Baldry, The Unity of Mankind in Greek Throught, Cambridge 1964; H. Dörrie, "Die Wertung der Barbaren im Urteil der Griechen", Antike und Universalgeschichte. Festschrift Hans Erich Stier, Münster 1972, S. $146 \mathrm{ff}$.; R. Müller, "Hellenen und 'Barbaren' in der griechischen Philosophie", Menschenbild und Humanismus der Antike, Leipzig 1980, S. $111 \mathrm{ff}$.

${ }_{2}$ Zur ethnographischen Tradition und ihrer Einbettung in umfassendere Zusammenhänge der antiken Wissenschaftsgeschichte vgl. K. E. Müller, Geschichte der antiken Ethnographie und ethnologischen Theoriebildung, 1 - 2, Wiesbaden 1972/80 (Studien zur Kulturkunde 29/52). 
den im Perserkrieg zutage tretenden grundlegenden Unterschied in der politischen Ordnung von Griechen und Persern (VII 102, 1). An anderer Stelle gibt es aber auch bereits Vorzeichen einer künftigen Entwicklung bestimmter Bewertungskriterien, wenn postuliert wird, die Griechen seien von alters her «klüger» gewesen als die Barbaren $(I 60,3)$. Im Gefolge der Perserkriege hat sich denn auch die Hellenen-BarbarenAntithese herausgebildet: die Auffassung von der prinzipiellen, da "naturbedingten" Überlegenheit der Griechen über alle anderen Völker, die mit philosophisch-theoretischen Mitteln abzustützen, antike Philosophen und Gelehrte über Jahrhunderte nicht müde wurden.

Von einem, dem entscheidenden dieser theoretischen Instrumentarien, das bis in die Neuzeit hinein, wenn auch mit veränderter Zielsetzung, lebendig blieb, muß hier vor allem die Rede sein: der Klimatheorie. Am Anfang stand der die frühe griechische Wissenschaft auszeichnende ätiologische Geist, das Fragen nach den Ursachen, das diese Wissenschaft auf ihre außerordentliche Höhe führte. Bei der Klimatheorie verband sich dieser Geist jedoch schon früh mit Motiven, die aus dem Bereich der Politik kamen: dem nach dem Sieg über die Perser gewonnenen Selbstverständnis einer politischen und kulturellen Überlegenheit und daraus abgeleiteten Herrschaftsansprüchen.

Schon das erste uns erhaltene Zeugnis einer umfassenden klimatheo-

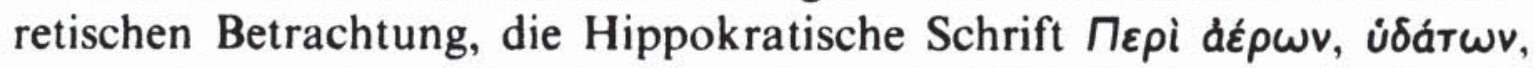

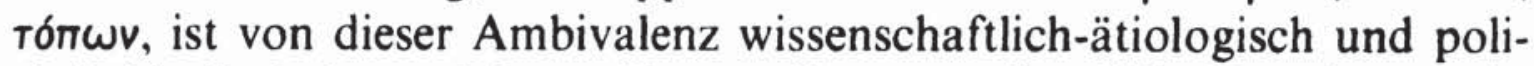
tisch-ideologisch motivierter Fragestellung geprägt. Wie Herodot macht der Autor der Schrift den Vergleich Asien - Europa zum Drehpunkt seiner Betrachtung, ergänzt durch eine Nord-Süd-Achse, die durch die Einbeziehung Skythiens in Europa, Ägyptens und Libyens in Asien zur Geltung kommt. Während in der Nord-Süd-Perspektive Asien mit seiner Mittellage zwischen extrem kaltem Norden und extrem heißem Süden den Vorrang zu haben scheint, entsteht durch die Gegenüberstellung von Europa und Asien ein anderes Bild. Während Asien als Mitte zwischen Nord und Süd günstigere Bedingungen für Flora und Fauna und die körperliche Gestalt der Menschen schafft, prägt der Grad der Klimaschwankungen die seelisch-geistige Struktur: Die Menschen Asiens seien infolge des dort herrschenden großen klimatischen Gleichmaßes passiv, großen Anstrengungen abgeneigt und feige, weshalb sie sich leicht in das Joch asiatischer Despotie fügten. Dagegen verursachten starke Klimaschwankungen in Europa einen Menschentyp, der von geistiger Beweglichkeit, Standhaftigkeit und Tapferkeit geprägt sei, weshalb dort die politische Autonomie zum wesentlichen Merkmal wurde (12, $1 \mathrm{ff}$.; 16, $1 \mathrm{ff}$.; 23, $1 \mathrm{ff}$.). Die Schlußfolgerungen münden in 
die zu eben dieser Zeit sich herausbildende politisch-ideologische Argu-

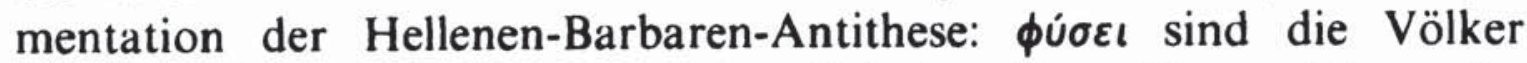
Asiens dafür prädestiniert, unter politischer Despotie zu leben; der vónoৎ wirkt diesen klimatischen Bedingungen analog und fördert $\mathrm{zu}$ sätzlich die entsprechenden Eigenschaften ${ }^{3}$.

Welche Bedeutung die Hellenen-Barbaren-Antithese, die im 5. und 4. Jh. gewissermaßen ihre klassische Gestalt gewann, für die Entwicklung des griechischen Denkens hatte, kann hier nur an den beiden bedeutendsten Ausprägungen, die wir bei Platon und Aristoteles finden, gezeigt werden. Dabei müssen wir vorausschicken, daß die Entwicklung der sozialphilosophischen und der politischen Theorie im 5. und 4. Jh. eine größere Vielfalt aufweist, als wir hier auch nur andeuten können. In gegenläufiger Tendenz zum Hellenen-Barbaren-Schema gab es bei ei-

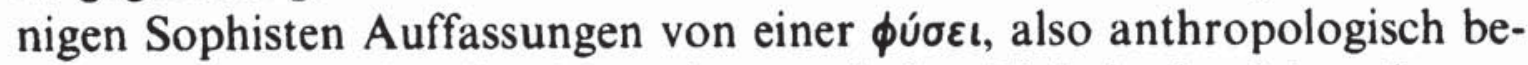
dingten Gleichheit aller Menschen und der Einheit des Menschengeschlechts ${ }^{4}$. So bedeutsam sie uns heute geistesgeschichtlich erscheinen, dürfen sie doch in ihrer Wirkung im zeitgeschichtlichen Bedingungsgefüge nicht überschätzt werden, da sie auf einen kleinen Kreis von Theoretikern beschränkt blieben. Aber auch bei Platon und Aristoteles kann von einer einschichtig-linearen Verwendung des Schemas nicht die Rede sein.

Platon hat für die gesellschaftlichen und politischen Strukturen des alten Persiens und Ägyptens und für die kulturellen Leistungen der Ägypter große Bewunderung gehegt ${ }^{5}$. In beiden Fällen geht es freilich um die Geschichte, nicht um die empirische Gegenwart dieser Völker. Die kulturellen Leistungen der Ägypter, mit deren hoher Wertschätzung Platon in der Tradition Herodots steht, werden vor allem im Hinblick auf wissenschaftliche Errungenschaften in der Mathematik und in der Astronomie gewürdigt. Das hat Platon nicht gehindert, der ideologisch motivierten Anwendung der Klimatheorie im Sinne der Hellenen-Barbaren-Antithese recht massiven Tribut zu zollen: Während Thraker und

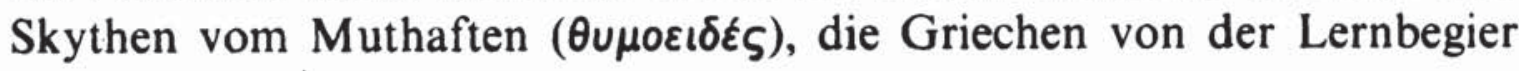

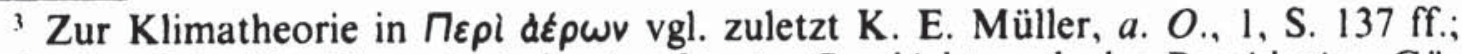
Katharina Schmidt, Kosmologische Aspekte im Geschichtswerk des Poseidonios, Göttingen 1980, S. 36 ff. (Hypomnemata 63).

${ }^{4}$ Vgl. Baldry, a. O., S. 42 ff.; R. Müller, "Zum Menschenbild der sophistischen Aufklärung», Menschenbild und Humanismus der Antike, S. $86 \mathrm{ff}$.

5 R. Müller, "Platon und der Orient. Zum Verhältnis von Hellenen und 'Barbaren'", Caucasica - Mediterranea. Kavkaz i Sredinomor'e, Tbilissi 1980, S. 139 ff. Von der älteren Literatur seien genannt: F. Weber, Platons Stellung zu den Barbaren, München 1904; J. Geffcken, "Platon und der Orient", Neue Jahrbücher für Wissenschaft und Jugendbildung 5, 1929, S. 517 ff.; K. Vourveris, Platon und die Barbaren, Athen 1938; J. Kerschensteiner, Platon und der Orient, Stuttgart 1945. 


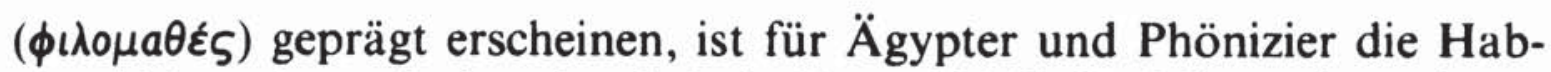

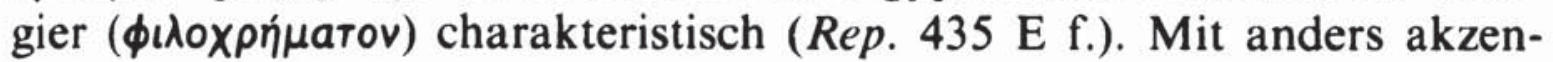
tuierten Inhalten scheint hier der Gegensatz von nördlichen und südlichen Determinierungen einerseits und den Vorzügen der Mittellage, die die Griechen geprägt haben, andererseits deutlich genug durch. Man muß hier die Gesetze ergänzend heranziehen, wo von den klimatischen Einflüssen und ihren Folgen noch deutlicher die Rede ist: nur den Griechen blieb die ooфía vorbehalten ( $\mathrm{Leg} .747 \mathrm{~B}$ ff.) ${ }^{6}$.

Auch Aristoteles ist weit entfernt, die bedeutenden Leistungen der orientalischen Kulturen und Staatswesen zu unterschätzen, wie viele Stellen seines Werkes zeigen. Für seine Stellung im Zusammenhang der Hellenen-Barbaren-Antithese ist jedoch vor allem die theoretische Begründung der Sklaverei wichtig. Die umstrittene Frage, ob sich Aristoteles in einem Satz der Politik mit dem Dichterwort $« \mathrm{Da}$ Griechen über Barbaren herrschen, ist billig» unmittelbar identifiziert und damit die Gleichung von Barbar und Sklave auch explizit herstellt, ist freilich

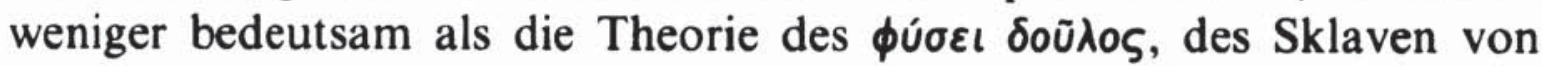
Natur, in ihrer Gesamtheit (Pol. I 3, 1253 b 1 ff.). Diese Theorie soll zeigen, daß es Menschen gibt, die potentiell von der Natur (auf Grund ihrer psycho-physischen Ausstattung) bestimmt sind, als Sklaven zu dienen, und da $\beta$ es ein gerechter Krieg ist, der dazu dient, Menschen dieser Art aus dem Zustand der potentiellen in den der realisierten Sklaverei zu überführen (Pol. I 8, 1256 b $15 \mathrm{ff}$. $)^{7}$. Auch hier findet die Klimatheorie Anwendung, wobei der mit Aristoteles' $\mu \varepsilon \sigma o ́ т \eta \varsigma$-Lehre so gut harmonierende Gedanke der geographischen Mittellage wiederum zum zentralen Kriterium wird: Die Völker der kalten Regionen im Norden und in Europa seien zwar mutig, wiesen aber einen Mangel an Intelligenz und Sachverstand für politische Organisation auf; die Völker Asiens seien durch Intelligenz und Sachverstand ausgezeichnet, aber feige und lebten daher in ständiger Knechtschaft. Die Griechen, die in der Mitte leben, vereinten in sich die Vorzüge beider, ohne ihre Schwächen zu teilen (Pol. VII 7, 1327 b 21 ff.).

Eine neue historische Situation ergab sich auch für unseren Zusammenhang aus der historischen Entwicklung in hellenistischer Zeit. Besonders die engere Symbiose der orientalischen Kulturen mit der griechischen schuf neue Ansatzpunkte für die Auseinandersetzung mit dem

${ }^{6} \mathrm{Vgl}$. R. Müller, Platon und der Orient, S. $142 \mathrm{ff}$, über die $\sigma o \phi i ́ n$ bei Herodot und die Fortführung Platonischer Gedanken in der Epinomis.

$7 \mathrm{Vgl}$. H. Klees, Herren und Sklaven. Die Sklaverei im oikonomischen und politischen Schrifttum der Griechen in klassischer Zeit, Wiesbaden 1975, S. 181 ff. (Forschungen zur antiken Sklaverei 6). 
Barbarenproblem ${ }^{8}$. Neben konservativen Tendenzen in Akademie und Peripatos, aber auch bei Epikur, gab es die weit in die Zukunft weisende Menschheitsidee der frühen Stoa. Im Hinblick auf den sozialen und politischen Gleichheitsgedanken verharrte diese freilich in der Utopie und stellt so eine frühe Antizipation von Ideen dar, die erst Jahrhunderte später reale Bedeutsamkeit gewinnen sollten. Für unser Thema des Herrschaftsdenkens gewann höhere praktische Relevanz die Entwicklung, die die Stoa im politisch-kulturellen Verschmelzungsprozeß von Griechenland und Rom erfuhr ${ }^{9}$.

Wie also steht es mit Rom? Wichtig sind hier die Ansprüche, die Rom mindestens seit spätrepublikanischer Zeit auf eine durch den Willen der Götter vorherbestimmte Herrschaft über die Völker erhoben hat. Die Frage ist, ob und in welcher Form die Philosophie zur theoretischen Stützung solcher Ansprüche beitrug ${ }^{10}$. Die sog. Mittlere Stoa hat, von Grundpositionen der frühen Stoiker sich weit entfernend, gerade in dieser Frage eine bemerkenswerte Annäherung an römische Denkstrukturen vollzogen, sicher nicht allein deshalb, weil ihre Repräsentanten Panaitios und Poseidonios auch in enge persönliche Beziehungen zu Vertretern der römischen Aristokratie traten ". Von Panaitios' relevanten Auffassungen wissen wir nicht viel, aber doch immerhin, daß er bereits den stoischen Gedanken der Gleichheit aller Menschen in Anlehnung an die traditionelle Klimatheorie in Frage gestellt hat. Wie bereits Platon im Timaios ( $24 \mathrm{C}$ f.) vertrat er die Auffassung, daß ein ausgeglichenes Verhältnis der Jahreszeiten speziell Attika prädestiniert habe, "vernünftige Männer» hervorzubringen (Fr. 76 van Straaten). Hier geht es also immer noch um die Griechen und ihre privilegierte Stellung, und man fragt sich, wie lange in der gewandelten Situation des römischen Weltreichs solche Positionen aufrechterhalten bleiben konnten. Damit kommen wir zu einem Gegenstand, der, seit längerem umstritten, im Lichte neuerer Entwicklungen in der Forschung ungleich deutlichere Konturen zeigt, als das noch vor wenigen Jahren abzusehen

${ }^{\rtimes}$ Zur Entwicklung in hellenistischer Zeit vgl. R. Müller, «Hellenen und 'Barbaren' in der griechischen Philosophie», S. $121 \mathrm{ff}$.

${ }^{9}$ Zum Gesamtkomplex vgl. The Interrelations of Greek and Roman Civilization. Concilium Eirene XVI. Proceedings of the $16^{\text {th }}$ International Eirene Conference, Prague 31.8. - 4.9.1982. Edited by Pavel Oliva and Alena Frolíková, Prag 1982.

${ }_{10}$ R. Müller, "Kosmos und Imperium. Zur römischen Ideologiegeschichte der späten Republik und der Kaiserzeit". Ideologie und Geschichte im alten Rom. Dem Wirken Werner Hartkes gewidmet, SB der Akademie der Wissenschaften der DDR. Gesellschaftswissenschaften, Jg. 1987, Nr. 14/G, Berlin 1987, S. 8 ff.

"Zur Rolle der Mittleren Stoa in der theoretischen Rechtfertigung der römischen Expansion W. Capelle, "Griechische Ethik und römischer Imperialismus», Klio 25,1932 , S. 86 ff. 
war. Es geht um Elemente in der politisch-historischen Theorie des Poseidonios.

Schon seit längerem hat ein Poseidonios-Fragment starke Beachtung gefunden, in dem Denkstrukturen der Platonisch-Aristotelischen Theorie eine merkwürdige Wiederbelebung erfahren. In Anknüpfung an eine in Griechenland offenbar seit langem geführte Diskussion über neben der Sklaverei existierende Abhängigkeitsverhältnisse und in wohl direktem Anschluß an Platon, der sich an dieser Diskussion beteiligt hatte, lenkt Poseidonios die Aufmerksamkeit auf den kleinasiatischen Stamm der Mariandyner, die sich freiwillig unter die Herrschaft und unter den Schutz der megarischen Kolonie Herakleia begeben hätten. Geleitet von der Einsicht in eine naturbedingte Schwäche ihrer geistigen Fähigkeiten

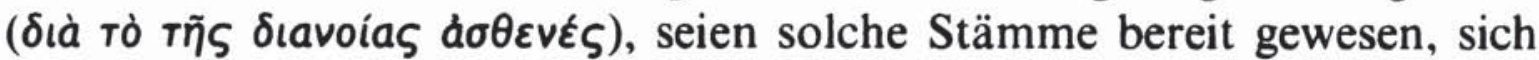
in den Dienst der geistig Überlegenen zu stellen, um dafür deren Fürsorge zu erfahren (Fr. 147 Theiler). Diese für einen Stoiker erstaunliche, sich vom Gedanken der natürlichen Gleichheit aller Menschen weit entfernende Begründung politischer Herrschaftsverhältnisse auf biologischer Ungleichheit hat eine sehr unterschiedliche Bewertung gefunden ${ }^{12}$.

Eine Möglichkeit, das Fragment aus seiner isolierten Stellung zu befreien, ergibt sich, wenn wir den Bezug auf eine Passage im 3. Buch von Ciceros De re publica herstellen, auf den K. Reinhardt verweist. Natürlich geschieht das im Bewußtsein der komplizierten Quellenfragen, die sich ergeben, wenn man hier nicht an eine punktuelle Bezugnahme, sondern an eine umfassendere Form der Abhängigkeit denkt. Im Kontext von Ciceros berühmter Auseinandersetzung mit Karneades' Kritik an der römischen Politik wurde, wie uns Augustin berichtet, im Namen der Gerechtigkeit verkündet, daß Menschen anderen Menschen, die über sie herrschen, dienten, sei deshalb gerecht, "weil solchen Menschen die Knechtschaft nützlich sei und es ihrem Nutzen diene, wenn die Herrschaft richtig ausgeübt wird... und es den Unterworfenen besser geht, weil es ihnen, noch nicht unterworfen, schlechter ging». Und nach der inhaltlichen Zusammenfassung als wörtliches Zitat: "Oder sehen wir nicht, daß von der Natur selbst immer dem Besten die Herrschaft zum größten Nutzen der Schwachen gegeben ist?... (De re publica III 36). Das naturrechtlich (ueluti a natura sumptum nobile exemplum) begründete Verhältnis zwischen dem "Besten» und den Schwachen dient also zur Rechtfertigung der römischen Herrschaft, wobei der Gedanke der Fürsorge des Besten für die Schwachen einen Bezug zum Mariandyner-

12 H. Strasburger, "Poseidonios on the Problems of the Roman Empire», JRS 55, 1965, S. $40 \mathrm{ff}$, warnt vor weitreichenden Verallgemeinerungen; anders K. Reinhardt, Art. "Poseidonios», RE, 43. Halbb., Stuttgart 1953, S. 825 f. 
Fragment des Poseidonios herstellt. Im Unterschied zu anderen Auffassungen kann es u. E. keinen Zweifel an der insgesamt stoischen Prägung des gesamten Abschnitts geben. Die spezifische Form einer naturrechtlichen Begründung spricht eine deutliche Sprache. Darauf, daß es sich um einen Stoizismus Poseidonischer Prägung handelt, weisen eine Reihe weiterer Bezüge, die hier nur in Kürze angedeutet werden können ${ }^{13}$.

Der Gedanke der Herrschaft des Besten zum Nutzen der Schwachen ist auf gewissermaßen innerstaatlicher Ebene ein Hauptelement der Poseidonischen Konzeption von der Frühgeschichte der Menschheit, wie sie Senecas Brief 90 überliefert. Für Poseidonios spricht auch eine merkwürdige Verschiebung, die ein ursprünglich Aristotelischer Gedanke gefunden hat. Während Aristoteles das Verhältnis Herr - Sklave durch die Analogie von Seele und Körper veranschaulicht hatte, wird jetzt analog dazu von der Herrschaft der Weisheit als des besten Teils der Seele über die schwachen, triebhaften Seelenteile gesprochen (De re publica III 37). In historisch gesehen aufschlußreicher Weise rückt anstelle der Sklaverei das Verhältnis zwischen herrschenden und unterdrückten Völkern in den Vordergrund. Die Denkkategorien sind insgesamt die der Platonisch-Aristotelischen Begründung der Sklaverei, aber ihre Anwendung erfolgt, angemessen der veränderten historischen Situation, im Sinne der Herrschaft eines Volkes über andere Völker. Dafür dient die Verschiebung vom Verhältnis Seele - Körper auf die Relation optima pars animiuitiosae imbecillaeque partes: Herrschaft des besten Elements zugunsten der schwachen. Wie stark aber das Schema Herr - Sklave immer noch durchschimmert, sowohl im Poseidonios-Fragment wie bei Cicero, zeigt die Berufung auf die Fähigkeit zu voller Selbstverantwortung als entscheidendes Kriterium. "Wer von Natur nicht sich selbst, sondern einem anderen gehört, aber ein Mensch ist, ist ein Sklave von Natur» lautet die berühmte Aristotelische Definition (Pol. I 4, 1254 a 14 ff.). Das Argument einer Unfähigkeit zur Selbstverantwortung wird nun auf ganze Stämme oder Völker ausgedehnt: Anwärter für ein Abhängigkeitsver-

${ }^{13}$ Es ist hier nicht möglich, die komplizierten Quellenprobleme der Ciceronischen Schrift De re publica umfassend zu erörtern. In der neueren Untersuchung von $\mathrm{H}$. Th. Johann, Gerechtigkeit und Nutzen. Studien zur ciceronischen und hellenistischen Naturrechts- und Staatslehre, Heidelberg 1981, werden gute Gründe für einen stärkeren Einfluß des Poseidonios auf diese Schrift vorgebracht. Zu unserer Stelle vgl. S. 363 ff. Wichtige Argumente für die Zurückführung von De re publica III 36 f. auf die Mittlere Stoa bereits bei Capelle, a. O., der aber, der traditionellen Zuweisung an Panaitios folgend, für Poseidonios in dieser Frage nur einen Anschluß an seinen Lehrer angenommen hatte. Die von Johann erörterten Gesichtspunkte, die für unmittelbare Herkunft der Gedanken von Poseidonios (in der Substanz, wenn auch vielleicht nicht in der Formulierung) sprechen, finden in den hier dargelegten Zusammenhängen Unterstützung. 


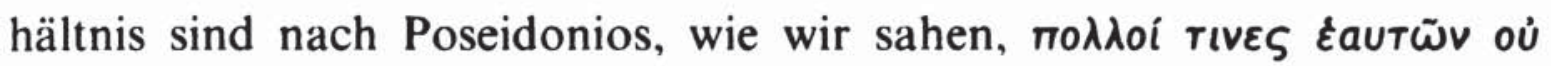

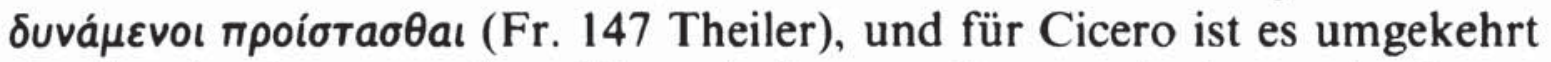
ein Ausdruck ungerechter Herrschaft: cum ii sunt alterius, qui sui possunt esse (De re publica III 37).

Nun ordnen sich diese überraschenden Gemeinsamkeiten der politisch-theoretischen Argumentation bei Cicero und Poseidonios in übergreifende Zusammenhänge ein, die durch neueste Forschungen zum Geschichtsbild des Poseidonios deutlicher hervorgetreten sind, ohne daß man, erstaunlicherweise, zu jenen bedeutsamen Elementen politischer Theorie einen Bezug hergestellt hätte.

In einer Untersuchung zum Poseidonischen Geschichtsdenken ist es gelungen nachzuweisen, was früher schon vielfach vermutet worden war: da $\beta$ im Kern auf Poseidonios bestimmte Texte zurückgehen, in denen aus klimatheoretischen Erwägungen Schlußfolgerungen für die Rolle von Völkern im Geschichtsprozeß gezogen werden ${ }^{14}$. Für unseren Zusammenhang bedeutsam ist an erster Stelle eine Partie bei Vitruv, die in diesem Rahmen seit langem Beachtung gefunden hat (VI 1, 3 ff.). Ein Begriff der besten, da negative Extreme ausschließenden Mitte zwischen Norden und Süden wird bei Vitruv nicht nur im Hinblick auf die körperliche Beschaffenheit (VI 1, 3-8), sondern auch auf die Mentalität konstatiert (VI 1, 9-12): Da bei den Völkern des Nordens höchste Tapferkeit durch die Verbindung mit einem trägen Geist, bei den Völkern im Süden ein scharfer Verstand durch Feigheit beeinträchtigt wird, müssen beide als unzulänglich erscheinen. Tapferkeit ohne Verstand führt zum unbedachten Angriff; scharfer Verstand und Erfindungsgabe bleiben ohne Mut unwirksam. Die Römer als Volk der Mitte des ganzen Erdkreises (10), Italien in der Mitte zwischen Norden und Süden (11) bricht durch Klugheit den kriegerischen Mut der (nördlichen) Barbaren, durch Tapferkeit die verschlagenen Pläne der Südvölker. Die Konsequenz ist ein von der göttlichen Vorsehung verbürgter Anspruch auf Weltherrschaft: Ita diuina mens ciuitatem populi Romani egregia temperataque regione conlocauit, uti orhis terrarum imperii potiretur (11). Dürfen wir diese neue Lokalisierung der Mitte und die aus ihr abgeleitete Schlußfolgerung bereits auf Poseidonios zurückführen?

Die Frage, die K. Schmidt mit großer Zurückhaltung offen halten möchte, scheint durch die oben aufgezeigten Bezüge zur politischen Theorie des Poseidonios, wie sie sich bei Cicero spiegelt, einer positiven Antwort wesentlich näher zu kommen. Gewiß haben wir zunächst nur eine вüкрато५-Zone im Sinne eines Klimagürtels zwischen Norden und

${ }^{14}$ K. Schmidt, a. O., S. $26 \mathrm{ff}$. 
Süden anzunehmen. Aber die Autorin hat schon mit Recht darauf hingewiesen, da $\beta$ unter unterschiedlichen Gesichtspunkten verschiedene Abschnitte des Gürtels in den Mittelpunkt wertender Aussagen rücken konnten (so bei Vitruv VI 1, 6 media Graecia im Hinblick auf die Tonhöhe der Stimmen). Wie also steht es mit den Konsequenzen für die Ausübung politischer Herrschaft?

Hier ist nun der kurze klimatheoretische Abschnitt in der Italiendarstellung im 6. Buch Strabons (VI 4, 1), von K. Schmidt für Poseidonios gesichert ${ }^{15}$, von großer Bedeutung. Es wird nämlich den bisherigen Argumenten, die aus der Nord-Süd-Achse gewonnen sind, ein weiterer Aspcñt hinzugefügt, der sich aus einer west-östlichen Mittellage ergibt. Italien hat nicht nur durch seine lange Nord-Süd-Ausdehnung größtmöglichen Anteil am klimatisch günstigsten Bereich der Oikumene, sondern ist in der Mitte ( $\left.\varepsilon^{\prime} v \mu \varepsilon \delta \omega\right)$ zwischen den "größten Völkern" (im Westen, gemeint sind wohl Kelten und Iberer) einerseits und «Griechenland und den reichsten Gebieten Asiens" ander :rseits schon durch diese Mittellage und "weil es die umliegenden Gebiete an Leistungskraft und

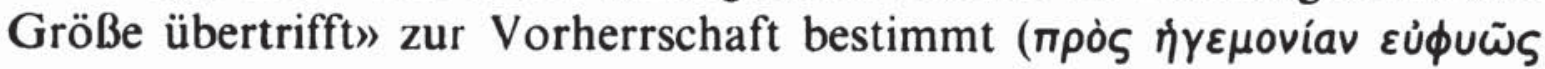

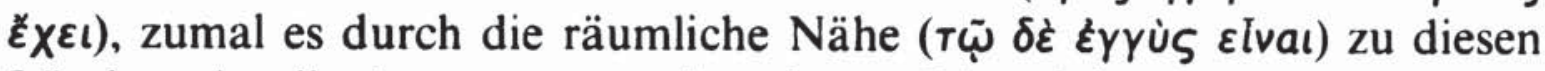
Ländern in die Lage versetzt ist, deren Dienstleistungen entgegenzunehmen.

Der gedankliche Zusammenhang scheint lückenlos: Was (bei Vitruv) der Nord-Süd-Bezug nicht mit letzter Sicherheit zu erweisen vermag, wird bei Strabon durch die zusätzliche Einführung der Ost-West-Achse zu weitgehender Wahrscheinlichkeit erhoben: daß Poseidonios mit klimatheoretischer Begründung das durch die historische Entwicklung hinfällig gewordene Hellenen-Barbaren-Schema zugunsten eines römischen Anspruchs auf Vorherrschaft über die Völker ersetzt hat.

Wie nun ordnet sich ein solcher Anspruch in Poseidonios' Geschichtsbild ein? Einerseits entspricht es der Poseidonischen Konzeption von der Einheit der Universalgeschichte, wie sie K. Reinhardt aus dem Proömium zum Werk Diodors erschlossen hat ${ }^{16}$. Diese wiederum ist eingebunden in die Auffassung von der Verwandtschaft und Gemeinschaft der Götter und Menschen, durch die die politischen Organisationsformen zur kosmischen Ordnung in Beziehung gesetzt werden, wie in Ciceros Staatsschrift (De re publica III $32 \mathrm{ff}$.) ausgeführt ist. Daß es sich hier um Poseidonische Denkstrukturen handelt, wird durch die mit Sicherheit von diesem Philosophen beeinflußte Partie in De off. I 153 nahegelegt.

${ }^{15}$ Ebenda S. $60 \mathrm{ff}$.

$16 \mathrm{Vgl}$. K. Reinhardt, Poseidonios, München 1921, S. 32 f. 
Poseidonios' theoretische Rechtfertigung eines römischen Herrschaftsanspruchs steht nicht im Widerspruch zu seiner Kritik an römischen Herrschaftspraktiken einerseits und seinem Verständnis für die Eigenart von Barbarenethne, wie es uns in seiner Darstellung der Kelten, Iberer, Ligurer u. a. Völker begegnet ${ }^{17}$. Poseidonios hat, wie wir sahen, den Anspruch auf Herrschaft an eine Fürsorgepflicht gebunden, die dem Stoiker den Weg zur Abwendung vom ursprünglichen stoischen Gleichheitsgedanken erleichtert haben mochte. Sie steht auch nicht a priori im Widerspruch zu Poseidonios' Dekadenzkonzeption, da der Philosoph und Historiker zwar von einer bevorzugten Disposition des römischen Volkes zur Herrschaft ausgehen, deren Verwirklichung aber in seinem Geschichtswerk durchaus kritisch an den Taten und den Ergebnissen römischer Politik messen konnte ${ }^{18}$.

Daß Poseidonios aus subjektiver Überzeugung von einer Verpflichtung der Römer zu gerechter Herrschaft zu sprechen und darin eine Vorbedingung für die Erfüllung ihrer historischen Mission zu sehen vermochte, zeigt die moralisierende Art seiner Geschichtsbetrachtung in einem anderen Zusammenhang: in seinem Verhältnis zum Sklavenproblem. Poseidonios' berühmte Darstellung der Sklavenkriege in Sizilien (vgl. besonders Fr. $136 \mathrm{~b}$ - $\mathrm{f}$ Theiler) ist vor allem durch die Auffassung geprägt, daß das übermäßig grausame Verhalten einzelner Herren die Empörung und den Aufstand ausgelöst habe und daß ein maßvoller, von Humanität geprägter Umgang mit den Sklaven bewirken könne, da $\beta$ derartige Erscheinungen vermieden werden.

In der Spannung zwischen Poseidonios' von echtem wissenschaftlichem Verständnis für die Eigenart der Barbarenvölker bestimmten ethnographischen Darstellungen und dem Tribut, den auch er dem Prinzip einer Ungleichheit der Völker "von Natur" gezollt hat, spiegelt sich noch einmal der Zwiespalt, der das Verhältnis der meisten antiken Philosophen zu den Barbaren bestimmt: die Diskrepanz zwischen einer vorurteilsfreien wissenschaftlichen Auseinandersetzung mit den Eigenarten und historischen Leistungen anderer Völker und einer ideologisch motivierten Unterstützung von Herrschaftsansprüchen des jeweiligen Zentrums über die Peripherie.

Die wirklichen oder scheinbaren Widersprüche in der Theorie des Poseidonios verweisen auf umfassendere Zusammenhänge der historischen Realität: auf die Spannung im Verhältnis von Zentrum und Peripherie, die gerade auch die Pax Romana in ihrem Wesen bestimmt hat:

17 Vgl. J. Malitz, Die Historien des Poseidonios, München 1983, SS. 96 ff., 424 ff.

is H.-Th. Johann, a. O., S. 369 f.; K. Schmidt, a. O., S. $103 \mathrm{f}$. 
Förderin des historischen Fortschritts in einem Prozeß zu sein, der den Völkern der Peripherie fundamentale Anstöße für ihre Entwicklung gab, unter Bedingungen, die ihnen zugleich große Opfer abverlangten.

Reimar Múller 